\title{
The advantages of a spine coil over a torso coil in magnetic resonance imaging examination of the sternoclavicular joints
}

\author{
Artur Stefan Kusak ${ }^{1,2 B, D, E, F}$, Michał Tomasz Podgórski ${ }^{1 C, F}$, Piotr Grzelak ${ }^{1 A}$, Adam Kwapisz ${ }^{30, F}$ \\ 'Diagnostic Imaging Department, Polish Mother's Memorial Hospital Research Institute, Lodz, Poland \\ 2Magnetic Resonance Department, Medical Centers the Medici, Lodz, Poland \\ ${ }^{3}$ Clinic of Orthopaedics and Paediatric Orthopaedics, Medical University of Lodz, Lodz, Poland
}

\begin{abstract}
Purpose: There are two standard methods for an magnetic resonance imaging (MRI) examination of the sternoclavicular joints: with loop coils and the patient in the prone position, or with torso coils with the patient in a supine position. In some centres these joints are examined with the spine coil in a patient laying prone. There are no reports on the advantages of this method. Our hypothesis is that despite different MRI systems, application of a spine coil will improve examination quality.
\end{abstract}

Material and methods: Twenty-one healthy volunteers (10 female, 11 male, mean age 25 years) were randomised into three groups and scanned using three different MRI scanners (1.5T: Siemens Avanto, Philips Ingenia, 3.0T: Philips Achieva). Each volunteer was examined twice: using a standard protocol with a torso coil and with a spine coil, in prone position. The two groups were compared with regard to the intensity of motion artefacts using the $\chi^{2}$ test, and to the signal-to-noise ratio with the Wilcoxon signed-rank test.

Results: Application of a spine coil resulted in a significant decrease in the number of motion artefacts in all three planes (axial: $p=0.0004$; sagittal: $p<0.0001$; coronal: $p=0.0054$ ). Moreover, the signal-to-noise ratio was significantly increased with the application of a spine coil ( $28.6 \pm 8.6$ vs. $18.5 \pm 7.3$, respectively; $p=0.0002)$.

Conclusions: Application of a spine coil with the patient in a prone position is suitable for MRI evaluation of the sternoclavicular joints. It allows a higher signal-to-noise ratio and a lower intensity of motion artefacts to be obtained compared to a torso coil.

Key words: MRI, technique, protocol, sternoclavicular joints, torso coil, spine coil.

\section{Introduction}

The sternoclavicular (SC) joint is the only articulation that joins the upper extremity and the axial skeleton [1]. It can on rare occasions be affected by conditions such as septic arthritis, osteoarthritis, rheumatoid arthritis, trauma, SAPHO syndrome, and condensing osteitis [2-4]. However, due to the rarity of these conditions, evaluation of the SC joint creates a diagnostic challenge [1].
Despite being indispensable in the evaluation of most joints, magnetic resonance imaging (MRI) has yet to gain wide clinical acceptance in SC joint examination. This may be due to the wide availability of computed tomography (CT) examination, which is easier to perform and is subject to less respiratory blurring thanks to its shorter acquisition time [5]. However, application of the MRI scanner gives excellent spatial and contrast resolution, allows cartilage and soft tissues to be better characterised,

Correspondence address:

Dr. Artur Stefan Kusak, Diagnostic Imaging Department, Polish Mother's Memorial Hospital Research Institute, 281/289 Rzgowska St., 93-338 kódź, Poland,

e-mail:kusak.artur@gmail.com

Authors' contribution:

A Study design · B Data collection · C Statistical analysis · D Data interpretation · E Manuscript preparation · F Literature search · G Funds collection 
and is more sensitive to marrow oedema and fatty degeneration [6].

There are two standard methods for evaluation of the SC joint: one employs loop coils with the patient in the prone position, and the other uses torso coils with the patient in the supine position. As these two methods have major limitations; the aim of the study was to improve the examination technique in order to reduce the amount of motion artefacts and to increase the signal-to-noise ratio.

\section{Material and methods}

\section{Designing a new protocol}

The patient is positioned prone with the arms along the sides of the body. A cushion is placed under the forehead so the head lies straight in order to tense the sternocleidomastoid muscles equally. No straps are necessary to keep the head or chest in place. Although no additional cushion is needed for women to fit their breasts, for the sake of comfort, one is placed to fill the gap between the joint and the coil. The aiming laser is centred over the line connecting the spines of the scapulae. The reminder of the examination and its planning follows standard protocols described elsewhere [7].

\section{Comparison of examination protocols}

Twenty-one healthy volunteers (10 females and 11 males), with an average age of 25 years, were included in the study. They were randomised into three groups. Each individual was scanned twice using the following equipment:

- group 1 - examination with Siemens Avanto 1.5T using a spine matrix coil and matrix body coil: scan time for each coil $22 \mathrm{~min}$;

- group 2 - examination with Philips Achieva 3.0T using a total spine coil and torso coil: scan time for each coil $21 \mathrm{~min}$;

- group 3 - examination with Philips Ingenia 1.5T using a total spine coil in the table and torso coil: scan time for each coil $20 \mathrm{~min}$.

Standard sequences were used (TSE - T1W, T2W, PD, PD FatSat) in the axial, coronal, and sagittal planes

Table 1. Standard sequences parameters used in study

\begin{tabular}{|l|c|c|c|}
\hline Parameter & T1W & T2W & PD FatSat \\
\hline Matrix & $256 \times 256$ & $256 \times 256$ & $256 \times 256$ \\
\hline FoV Cor/Sag/Ax & $220 / 210 / 200$ & $220 / 210 / 200$ & $220 / 210 / 200$ \\
\hline Slice thickness & $3 \mathrm{~mm}$ & $3 \mathrm{~mm}$ & $3 \mathrm{~mm}$ \\
\hline Gap & $10 \%$ & $10 \%$ & $10 \%$ \\
\hline TR - range & $600-800$ & $3000-4000$ & $2500-3000$ \\
\hline TE - range & $15-25$ & $100-120$ & $25-30$ \\
\hline TSE & 3 & 18 & 8 \\
\hline NEX & 3 & 3 & 3 \\
\hline
\end{tabular}

(details are presented in Table 1). No breath holding was required.

Within each group, the intensity of the motion artefacts was assessed quantitatively and the signal-to-noise ratio qualitatively. The intensity of the motion artefacts was rated in all three planes (coronal, sagittal and axial) and categorised as: A quality (no/slight artefacts that allow to clearly differentiate borders of anatomical structures such as articular disc or stabilising ligaments); B quality (minor artefacts that still allowed for evaluation of anatomical conditions described above, but their margins were blurred); and C quality (major artefacts that did not allow for evaluation of anatomical conditions).

The signal-to-noise ratio (SNR) was calculated using Osirix MD software [8]. On axial scans, three square regions of interest were drawn: a 1000-pixel ROI covering two distal parts of both clavicles and part of the sternum, and two 500-pixel ROIs placed on the background air, just in front of the chest wall. The SNR was calculated according to the following formula:

$$
\mathrm{SNR}_{\text {single }}=0.655 \frac{S}{\mathrm{SD}_{\text {air }}}
$$

where $S$ is the mean signal in the larger ROI and $S D_{\text {air }}$ is the average of the standard deviations of the two smaller ROIs. The 0.655 factor was used because the (Gaussian) noise present on the raw data is centred about zero [8].

All examinations were evaluated by two experienced musculoskeletal radiologists ( 15 and 18 years of experience), who were blinded to the both type of examination protocol and type of scanner. Based on their assessment, the Cohen's kappa coefficient for interrater agreement was calculated. Following this, the examinations were re-evaluated by both specialists together, and the results obtained by consensus were used for further analysis.

The study protocol was approved by the local Bioethics Committee and was in accordance with the Helsinki Declaration of 1975, as revised in 2000. All participants gave their informed, written consent.

\section{Statistical analysis}

Statistical analysis was performed using Statistica 12 software (StatSoft Poland, Cracow, Poland). Generally, while a $p$-value $<0.05$ was considered significant, the Bonferroni correction was applied for multiple testing, and the level of significance was $<0.017$. For between-group comparison of intensity of the motion artefacts the $\chi^{2}$ test with dedicated corrections was applied. To evaluate interrater agreement in assigning to a particular category of motion artefacts, Kappa statistics were used. The normality of the data distribution was checked with the Shapiro-Wilk test. As the distribution was non-normal, comparisons of the SNR values of two scanning techniques obtained in the same patient were checked with the Wilcoxon signedrank test. 
Table 2. Number of quality categories for spine and torso coils according to scanning plane

\begin{tabular}{|l|c|c|c|c|c|c|c|c|c|}
\hline & \multicolumn{3}{|c|}{ Axial } & \multicolumn{3}{c|}{ Sagittal } & \multicolumn{3}{c|}{ Coronal } \\
\hline Coil & A & B & C & A & B & C & A & B & C \\
\hline Spine & 16 & 5 & 0 & 15 & 5 & 1 & 19 & 2 & 0 \\
\hline Torso & 2 & 14 & 5 & 0 & 9 & 12 & 10 & 9 & 2 \\
\hline$p$-value & \multicolumn{3}{|c|}{$0.0004^{*}$} & \multicolumn{3}{c|}{$<0.0001^{*}$} \\
\hline
\end{tabular}

*Significant differences
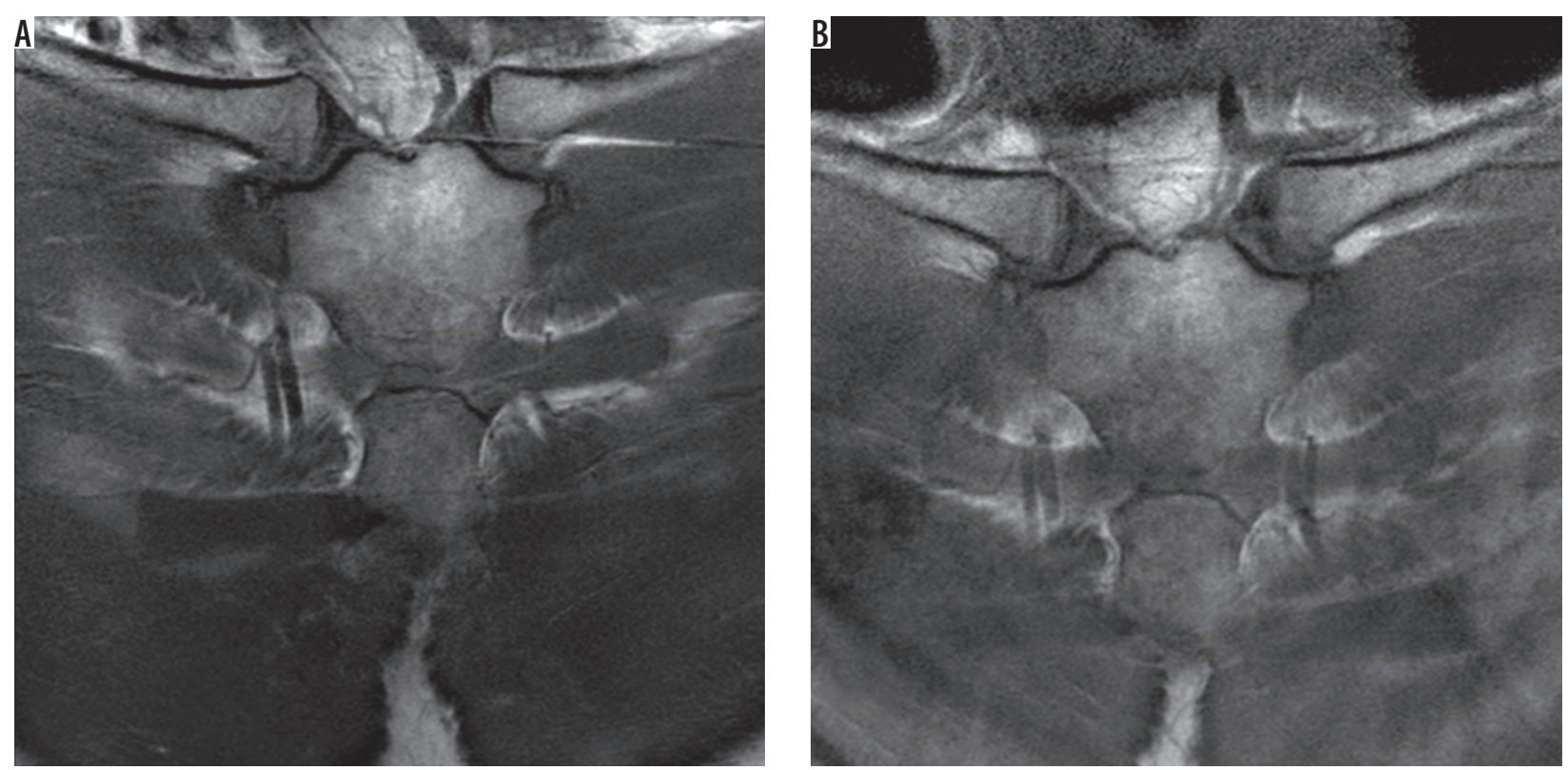

Figure 1. Examples of coronal scans of the sternoclavicular joints obtained from the Achieva 3T Scanner with A) spine and B) torso coils
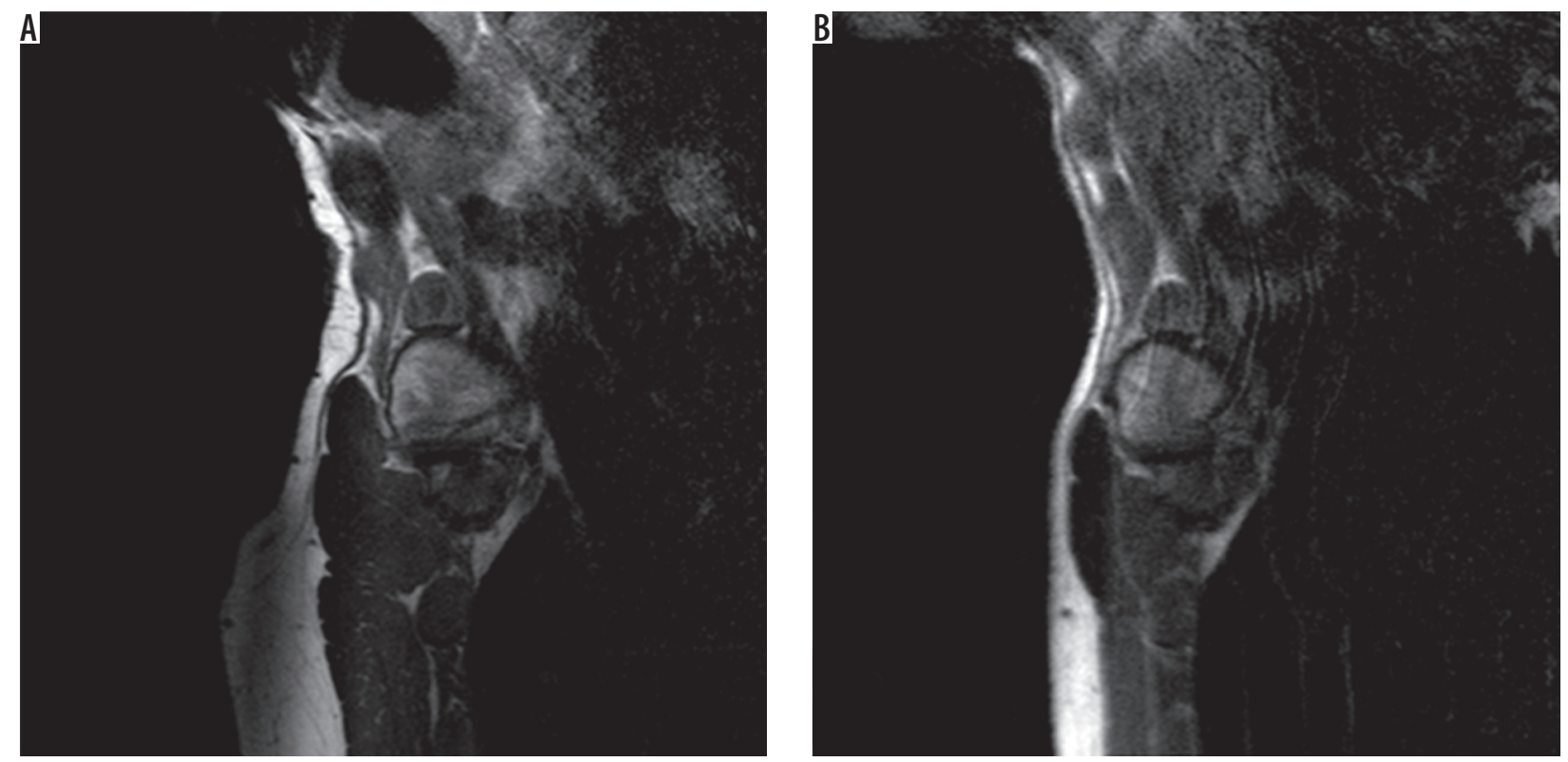

Figure 2. Examples of sagittal scans of the sternoclavicular joints obtained from the Ingenia 1.5T Scanner with A) spine and B) torso coils

\section{Results}

\section{Motion artefacts}

When the result for all scanners were combined, significantly fewer motion artefacts were found for the spine coil

than the torso coil in all three planes (Table 2, Figure 1). However, when analysed separately, only the axial (Figure 2) and sagittal (Figure 3) scans performed with the Achieva $3 \mathrm{~T}$ and Avanto 1.5T presented significantly fewer motion artefacts (data not presented). 

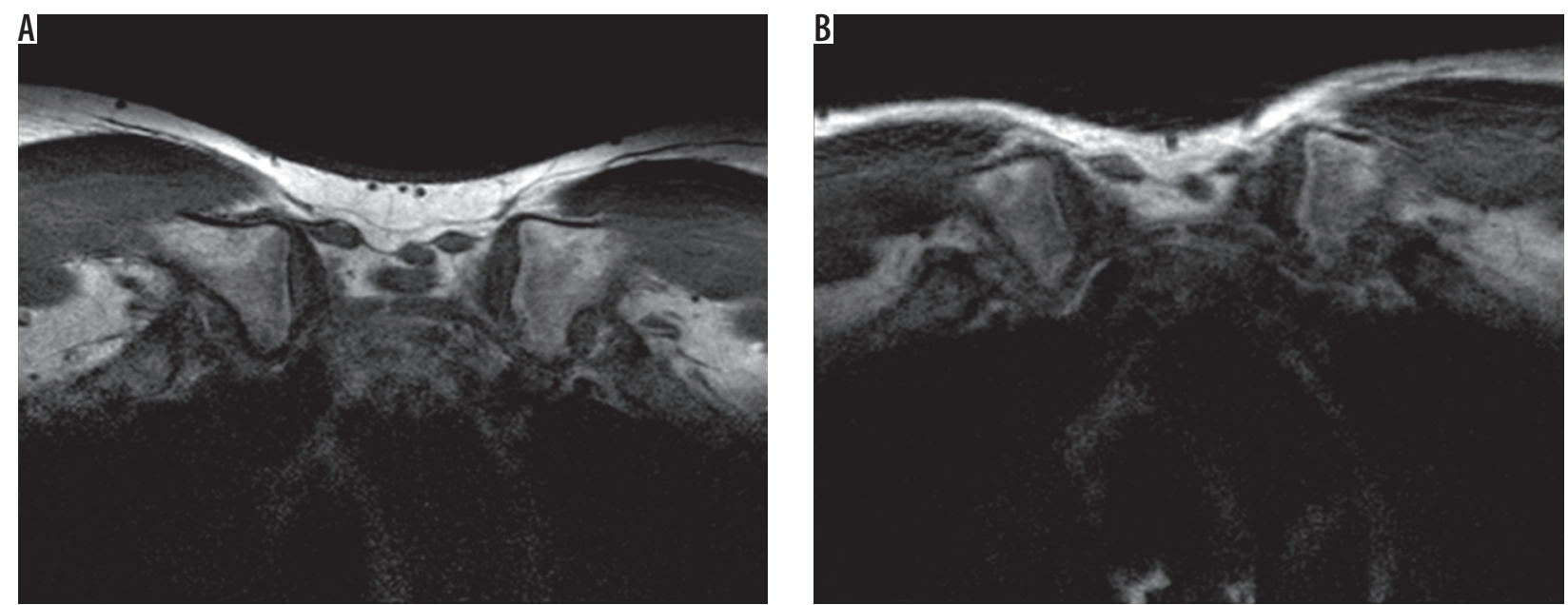

Figure 3. Examples of transverse scans of the sternoclavicular joints obtained from the Achieva 3T Scanner with A) spine and B) torso coils

Table 3. Signal-to-noise ratio (SNR) for spine and torso coil on different scanner

\begin{tabular}{|l|c|c|c|}
\hline \multirow{3}{*}{ Coil } & SNR [mean (SD)] \\
\hline Spine & $30.3(10.1)$ & $24.4(7.3)$ & $31.1(7.9)$ \\
\hline Torso & $24.8(8.0)$ & $14.8(3.0)$ & $16.1(5.8)$ \\
\hline$p$-value & 0.1763 & 0.0280 & $0.0156^{*}$ \\
\hline
\end{tabular}

*Significant differences

Kappa values for interrater agreement for axial, sagittal, and coronal planes were as follows: 0.64 (CI: 0.36-0.77) - substantial agreement; 0.87 (CI: 0.75-0.98) - almost perfect agreement; and 0.68 (CI: 0.38-0.88) - substantial agreement [9].

\section{Signal-to-noise ratio}

In general, the mean SNR was significantly higher for the spine coil than for the torso coil $(28.6 \pm 8.6$ vs. $18.5 \pm 7.3$, respectively; $p=0.0002$ ). However, when each scanner was analysed separately, the difference was significant only for the Achieva $3 \mathrm{~T}$ scanner (Table 3). For the other two scanners, there was a clear trend towards increasing SNR.

\section{Discussion}

Our study presents an efficient method for MRI examination of the SC joints based on the application of a spine coil. Not only is the scanning time comparable with routine methods, it is also associated with a greater signal-tonoise ratio and lower intensity of motion artefacts.

Although MRI examination is a valuable method for examining the sternoclavicular region [5], no consensus exists regarding the scanning protocol. Surface coils have been successfully used for virtualisation of the SC. Klein et al. reported clear visualisations of anatomical relations and pathological conditions [10] in this region with the use of a five-inch loop receive-only surface coil placed over the SC with the patient prone. However, the use of a loop coil presents some drawbacks: there is often a mismatch between coil size and patient size, loop coils are usually only single channel, which gives lower quality results, and the small size of the coil results in a restricted examination area. Additionally, when a surface coil is used with the patient in the supine position, the SC joint moves as the patient breathes, thus producing severe artefacts that are further worsened by vascular pulsation and swallowing [10].

However, more motion artefacts are produced when a torso coil is used, and its large field of view results in poorer spatial resolution. Although Aslam et al. [6] report obtaining excellent images of sternum and SC joints with the body coil and patients laying in supine position, the images given in the present study are of still higher quality.

Of course, the use of a breath-hold technique or respiratory gating may improve image quality when either coil is used. However, this method lengthens the scan time significantly and is not commonly used.

A literature search revealed one paper that describes an additional method for SC joint evaluation. Benitez et al. present images of a number of traumatic SC joint injuries obtained with the application of a two-part phased array shoulder coil placed directly over the SC joint and between the scapulae. However, all the presented images contained a significant number of motion artefacts [11]. Hence, because the most frequently injured structure was the articular disk and the SC joint should be assessed in all three planes, it is crucial that the scans are of optimal quality [5].

Our protocol facilitated increased SNR and reduced respiratory blurring, especially in planes that were the most affected with these artefacts. High image quality was obtained by positioning the patient as close to the coil as possible, which reduced the amount of free space between the patient and the coil (proper saturation). Moreover, the amount of respiratory movement was reduced by keeping the prone position to stabilise the SC 
joint. One unquestionable advantage of our method is its universalism: because spine coils are widespread in modern MRI equipment, it was possible to acquire satisfactory images using three different generations of MRI scanners. Moreover, this technique is faster than using breath-hold/respiratory gating techniques or following the standard procedure based on a torso coil, because patient alignment is easier.

This initial report has two main limitations. Firstly, the experimental group is quite small, and secondly, no comparison was possible between the use of a spine coil and a loop coil. However, as mentioned above, loop coil construction (single channel) is fundamentally compromised with lower scan quality, and loop coils are not used as often as torso or spine coils. Another potential limitation is that some patients, for example those after trauma or those with osteoarthritic changes in the neck or breathing problems, may be unable to lay in the prone position; however, this is only a potential problem that requires confirmation in a clinical setting.

\section{Conclusions}

This is an initial report that demonstrates the value of using a spine coil with the patient in the prone position for MRI evaluation of the SC joint. Despite different types of applied magnetic field (1.5T and 3T) and different generations of scanners, our technique allowed us to reduce the number of motion artefacts and to increase the signalto-noise ratio. Nevertheless, the usability of this method should be verified under clinical conditions on a larger group of patients.

\section{Conflict of interest}

The authors report no conflict of interest.

\section{References}

1. Manaster BJ, David AM, David GD. Musculoskeletal imaging the requisites. $4^{\text {th }}$ ed. Elsevier; 62. https://www.inkling.com/store/ book/musculoskeletal-imaging-manaster-may-disler-4th/

2. Jurik AG, Soerensen FB. Sternoclavicular joints. In: Imaging of the sternocostoclavicular region. Springer, Berlin, Heidelberg; 29-36. Available from: http://link.springer.com/10.1007/978-3-540-331483_3 (Acces: 28.08.2016).

3. Fischer W, Roemer F, Guermazi A. MRI - ESSENTIALS.COM MRI Publisher page 228. Available from: https://www.mri-publisher.com/

4. Kang BS, Shim HS, Kwon WJ, et al. MRI findings for unilateral sternoclavicular arthritis: differentiation between infectious arthritis and spondyloarthritis. Skeletal Radiol 2019; 48: 259-266.

5. Balcik BJ, Monseau AJ, Krantz W. Evaluation and treatment of sternoclavicular, clavicular, and acromioclavicular injuries. Prim Care 2013; 40: 911-923.
6. Aslam M, Rajesh A, Entwisle J, Jeyapalan K. MRI of the sternum and sternoclavicular joints. Br J Radiol 2002; 75: 627-634.

7. Elmaoğlu M, Çelik A. MRI Handbook. Springer US, Boston, MA 2012. Available from: http://link.springer.com/10.1007/978-1-46141096-6 (Access: 28.08.2016).

8. Firbank MJ, Coulthard A, Harrison RM, Williams ED. A comparison of two methods for measuring the signal to noise ratio on MR images. Phys Med Biol 1999; 44: N261-264.

9. Viera AJ, Garrett JM. Understanding interobserver agreement: the kappa statistic. Fam Med 2005; 37: 360-363.

10. Klein MA, Spreitzer AM, Miro PA, Carrera GF. MR imaging of the abnormal sternoclavicular joint - a pictorial essay. Clin Imaging 1997; 21: $138-143$

11. Benitez CL, Mintz DN, Potter HG. MR imaging of the sternoclavicular joint following trauma. Clin Imaging 2004; 28: 59-63. 